\title{
Características histológicas del ciclo reproductivo de Euvola ziczac (Linnaeus) (Pectinidae, Bivalvia) del litoral sur-sudeste del Brasil
}

\author{
Carlos Alberto Borzone ${ }^{1}$, Paulo Ricardo Pezzuto ${ }^{2} \&$ Yara Aparecida Garcia Tavares ${ }^{1}$ \\ ${ }^{1}$ Centro de Estudos do Mar, Universidade Federal do Paraná. Avenida Beira Mar, Caixa Postal 50002, 83255-000 Pontal do \\ Paraná, Paraná, Brasil.E-mail: capborza@ufpr.br; ytavares@ufpr.br \\ ${ }^{2}$ Centro de Ciências Tecnológicas, da Terra e do Mar, Universidade do Vale do Itajaí. Caixa Postal 360, 88302-202 Itajaí, \\ Santa Catarina, Brasil. E-mail: pezzuto@cttmar.univali.br
}

\begin{abstract}
Histological characteristics of the reproductive cycle of Euvola ziczac (Pectinidae, Bivalvia) from south-southern Brazil. Scallops were collected from beds at 30 to $40 \mathrm{~m}$ depth on the south-southern inner continental shelf of Brazil for histological gonadal characterization of the reproductive cycle. This hermaphroditic scallop was an important shellfish resource during 70-80 decade, with maximal annual landings of 8,845 tons, but now is depleted. Results shows that reproduction is continuous, with two major spawning periods, the first during end of summer and throughout autumn, and the second during winter and beginning of spring. A characteristic phenomenon of oocyte atresia, with maximal incidences in September is described.This phenomenon, followed by the mature oocyte resorption and new oocyte proliferation and maturation, was related to the lack of spawning stimulation. A semi-annual thermal cycle on the bottom water due to the South Atlantic Central Water intrusion seems to be the principal reproductive cycle controller. Fluctuations in landings registered since the beginning of the fishery may be partly related to oceanographic variations.
\end{abstract}

KEY WORDS. Oocyte atresia, reproduction, scallops, spawning stimulation.

Entre las diferentes especies de pectínidos que ocurren en la América del Sur y el Caribe, la vieira Euvola ziczac (= Pecten ziczac) (Linnaeus, 1758) ocupó un lugar destacado por su importancia comercial en la pesca extractiva (Salaya \& Penchazadeh 1979, Pezzuto \& Borzone 1997a), y por su posible potencial para la maricultura (Vélez et al. 1995). Esta espécie hermafrodita se distribuye desde el estado de la Carolina del Norte, en los Estados Unidos, hasta el estado de Santa Catarina, en el Brasil (Rios 1994). En el litoral brasilero existen bancos de Euvola ziczac en la plataforma continental interna entre las latitudes de $24^{\circ} 26^{\prime}$ y $26^{\circ} 30^{\prime}$ S, y en profundidades de 30 a $50 \mathrm{~m}$ (Pezzuto \& Borzone 1997a).

Oceanograficamente, la área ocupada por los bancos de vieira es conocida con el nombre de "Central South Brazil Bight", y se caracteriza por presentar una hidrología particular dominada por la intrusión, durante los meses del verano, de una masa de agua fría y rica en nutrientes denominada de "Agua Central del Atlántico Sur" (ACAS) (Matsuura 1986, Castro FILHO 1990, BoRzone et al. 1999). La dinámica de varias pesquerías demersales y pelágicas que ocurren en el área fueron relacionadas a esta particular condición oceanográfica (MaTsuURA 1996, Sunyé \& Servain 1998, Perez \& Pezzuto 1998).

Fue en esta área y durante los años 70, que comenzó uma importante extracción comercial de esta especie de vieira en el Brasil. El rápido incremento de la producción fue debido, por un lado, al aumento del valor del producto en el mercado internacional, y por otro, a la disminución de las capturas del camarón rosado, Farfantepenaeus paulensis (Pérez Farfante, 1967) y F. brasiliensis (Latreille, 1817), principal objeto de pesca de una flota industrial de arrastreros de tangones con sede en las ciudades de Santos, São Paulo e Itajaí, Santa Catarina. Los registros de desembarque se iniciaron en 1972, con un valor anual de 4,5 toneladas de vieira, mostrando los mayores valores en 1975 y 1980, con 3.799 y 8.845 toneladas anuales, respectivamente (Pezzuto \& Borzone 1997a). A partir de este último año, las capturas disminuyeron abruptamente para 10 a 20 toneladas anuales, siendo que de 1995 a 2000 los desembarques no pasaron de una tonelada.

A pesar de la importancia que tuvo el recurso en su época, los estudios biológicos realizados paralelamente a la pesquería fueron escasos, y con pocas excepciones (Амато \& Амато 1982, Morais \& KaI 1980), publicados en medios de divulgación restricta como informes técnicos internos (e.g. ZENGER et al. 1975).

Dentro de un proyecto mayor de reavaliación del actual estoque de vieiras y de su capacidad para sustentar el desarrollo de una pesquería, fue realizado un nuevo estudio histológico del ciclo reproductivo de Euvola ziczac. Este estudio tuvo como principales objetivos el de identificar variaciones ocasionadas por la intensa explotación pesquera anterior y su consecuente impacto en las densidades, así como también el de determinar las relaciones entre el ciclo reproductivo y las particulares características oceanográficas del área de ocupación de los bancos explotados. 


\section{MATERIAL Y MÉTODOS}

El material biológico estudiado fue colectado durante la realización de diversos cruceros oceanográficos entre marzo 1996 y mayo 1997, con el N/Pq Diadorim (CEPSUL/IBAMA) en los bancos denominados de São Francisco $\left(26^{\circ} 20,583^{\prime}\right.$ 's;

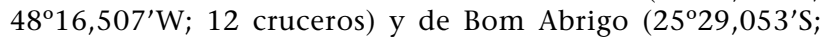
$47^{\circ} 37,451^{\prime} \mathrm{W} ; 6$ cruceros) (Fig. 1). Detalles de los cruceros pueden ser consultados en Pezzuto \& Borzone (1997b), Borzone \& Pezzuto (1997) y Pezzuto et al. (1998).

Colectas de sedimento y agua, junto con perfiles de temperatura y salinidad, fueron utilizadas para una detallada caracterización oceanográfica del área donde se encuentran los principales bancos de esta especie (Pezzuto et al. 1998, Borzone et al. 1999).

Para el estudio histológico, las gónadas de un número variable de individuos adultos (entre 9 y 20 individuos con altura $>55 \mathrm{~mm}$ ) de cada crucero fueron disecadas a bordo y fijadas en Bouin acuoso durante seis horas, para ser conservadas posteriormente en alcohol $70 \%$. En laboratorio fue realizado un corte transversal en la porción masculina y otro en la porción femenina de cada gónada, los cuales fueron procesados en una serie creciente de deshidratación, posteriormente diafanizados en xilol e incluidos en parafina. Cortes de $7 \mu \mathrm{m}$ de espesor fueron realizados con un micrótomo manual tipo Minot, y posteriormente teñidos con hematoxilina-eosina.

Después de un primer análisis de las láminas histológicas, fue confeccionada una escala de madurez del ciclo gametogénico, tomando como base escalas desarrollados para Chlamys tehuelchus (Orbigny, 1846) (Lasta \& Calvo 1978), Pecten maximus (Linnaeus, 1758) (Cochard \& Devauchelle 1993) y Argopecten circularis (Sowerby, 1835) (Villalejo-Fuerte \& Ochoa-Baez 1993), entre otros.

Además del tradicional diagnóstico por fases o estadios de desarrollo gametogénico (escala de madurez) realizado para cada individuo considerando independientemente el sector masculino y el sector femenino de la gónada, fueron realizados análisis cuantitativos en el sector femenino de 10 individuos de cada crucero realizado en el banco de São Francisco, con ayuda de un sistema de análisis digital de imágenes. Para cada crucero fueron obtenidas las siguientes medidas: área media interfolicular ( $\mathrm{n}=30)$; área media de los folículos $(\mathrm{n}=90)$; densidad media por folículo de: ovogonias, ovocitos íntegros (incluyendo a los ovocitos pre y vitelogenéticos, además de los maduros) y ovocitos atrésicos $(\mathrm{n}=900)$; diámetro medio de ovocitos íntegros seccionados por el núcleo $(300<\mathrm{n}<1700)$.

\section{RESULTADOS}

Los registros de temperatura mostraron una fuerte intrusión de la ACAS durante el período estudiado que determinó la formación de una fuerte termoclina y de un ciclo semianual en la temperatura del agua de fondo, con mínimos durante los meses de verano e invierno (Fig. 2). La salinidad mantuvo valores de 37 durante todo el año y en toda la columna del agua, con excepción de los meses de julio y agosto que mostraron una disminución para 27 debido a la entrada de aguas subantárticas (Fig. 3) (CAmpos et al. 1996, Borzone et al. 1999).

La escala de madurez del ciclo gametogénico para el sector femenino fue definida con las siguientes fases:

Maduración máxima: folículos de formas y dimensiones variadas, pero totalmente llenos de ovocitos vitelogénicos y maduros de gran tamaño $(60 \mathrm{a} 80 \mu \mathrm{m})$ y contornos regulares

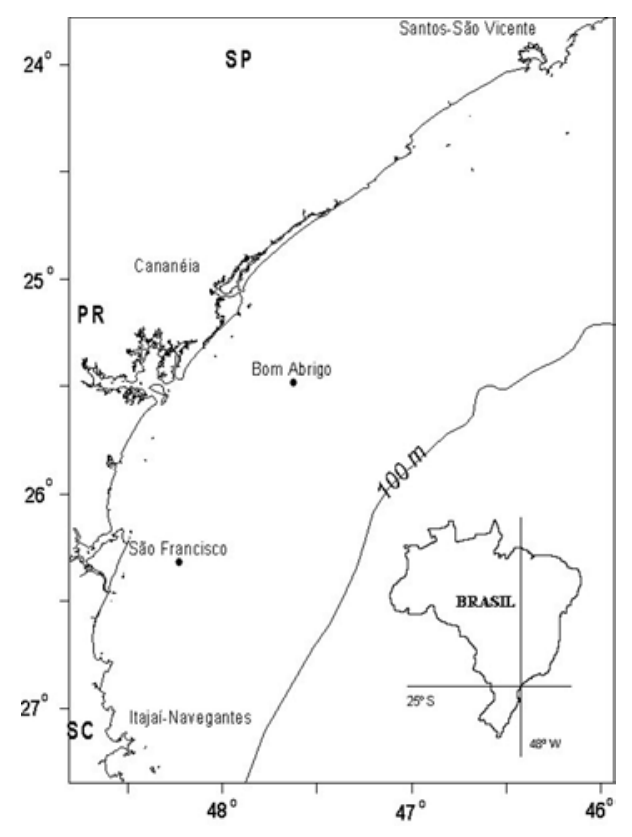

Figura 1. Locales de colecta $(\bullet)$ y principales puertos de desembarque de la vieira Euvola ziczac en Brasil. Estados de: (SC) Santa Catarina, (PR) Paraná, (SP) São Paulo.
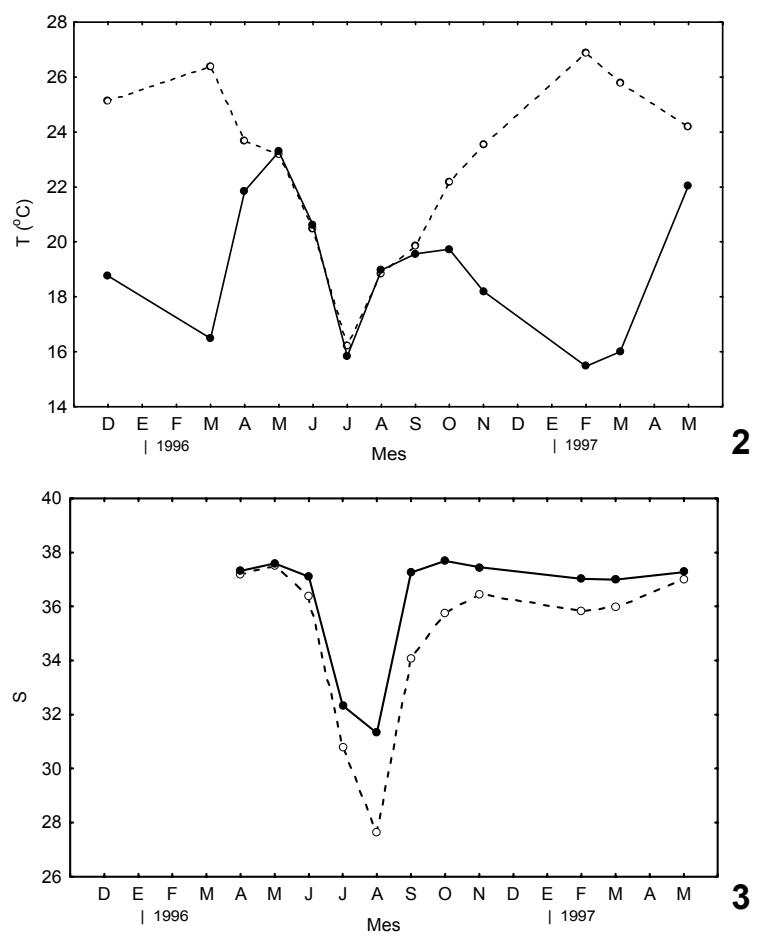

Figuras 2-3. Variación de la temperatura $\left({ }^{\circ} \mathrm{C}\right)(2)$ y de la salinidad (3) del agua de superficie (círculos blancos) y del fondo (círculos negros) en el banco de São Francisco. 

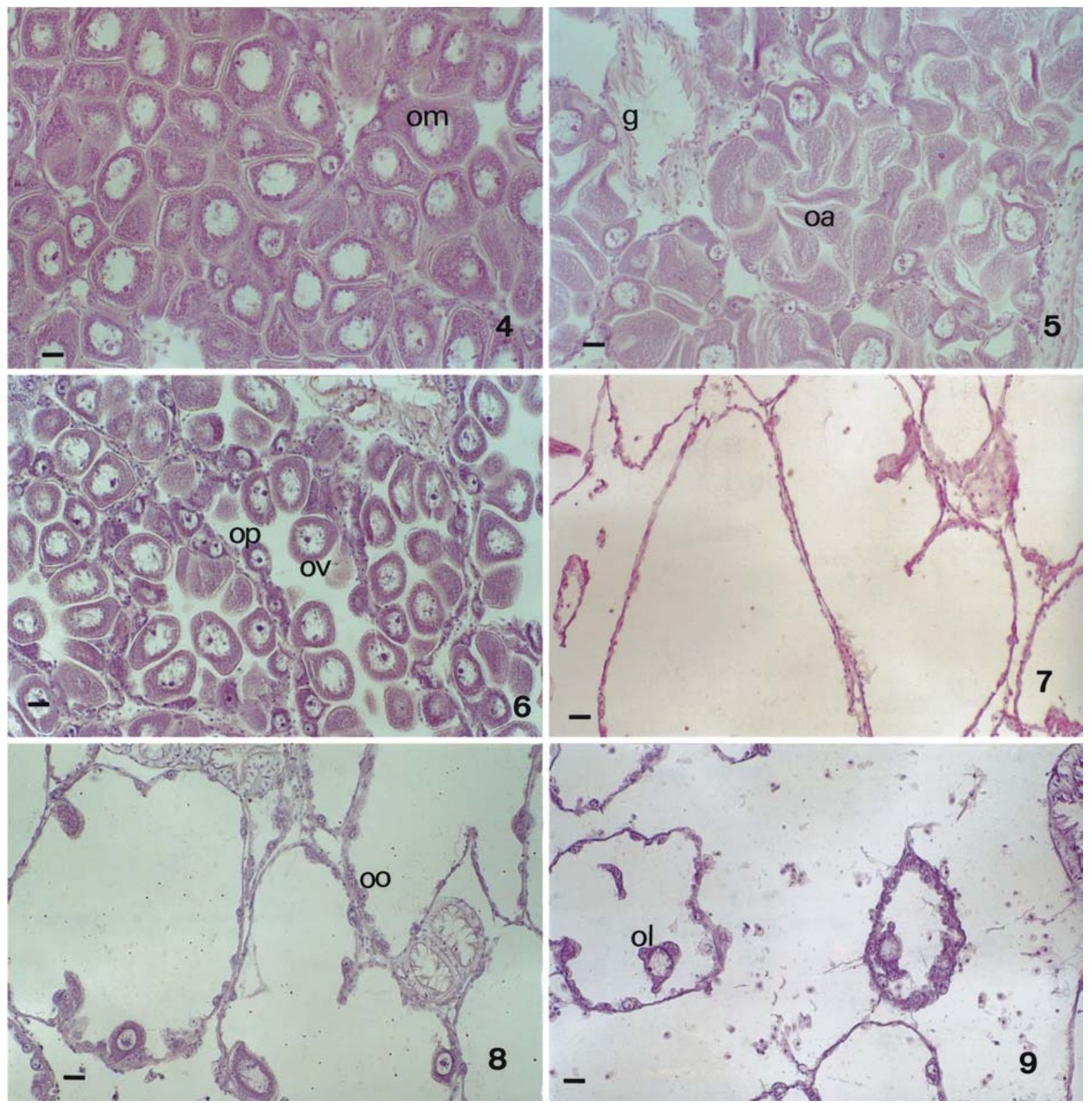

Figuras 4-9. Fases del desarrollo gametogénico del sector femenino de la gónada de Euvola ziczac: (4) maduración máxima; (5) maduración y atresia; (6) desove parcial con proliferación; (7) desove total; (8) desove total com proliferación; (9) reversión. (g) Gonoducto; (oa) ovocito atrésico, imagen de "pieza de rompecabezas"; (ol) ovocito en proceso de lisis celular; (om) ovocito maduro; (oo) ovogonia; (op) ovocito previtelogénico; (ov) ovocito vitelogénico. Escala: $50 \mu \mathrm{m}$.

poliédricos. En la mayoría de los ovocitos puede ser observado un espacio entre la membrana plasmática y el propio vitelo, característico de las estapas finales de la vitelogénesis. También podemos observar una pequeña cantidad de ovocitos previtelogénicos (20 a $30 \mu \mathrm{m}$ ) en la pared del folículo (Fig. 4).

Maduración y atresia: más de la mitad de los folículos mostrando una fuerte atresia en la totalidad o en la mayoría de los ovocitos. El núcleo de estos ovocitos pierde basofilia, el citoplasma se vuelve menos denso y el contorno del ovocito adquiere una forma irregular o ameboideo característica, configurando una imagen de "pieza de rompecabezas" ("jigsawpuzzle") (Fig. 5).

Desove parcial con proliferación: algunos folículos exhiben pocos o ningún ovocito maduro. Cuando presentes, estos se encuentran en lisis o reabsorción celular. Al mismo tiempo, otros folículos pueden estar llenos de ovocitos madu- 


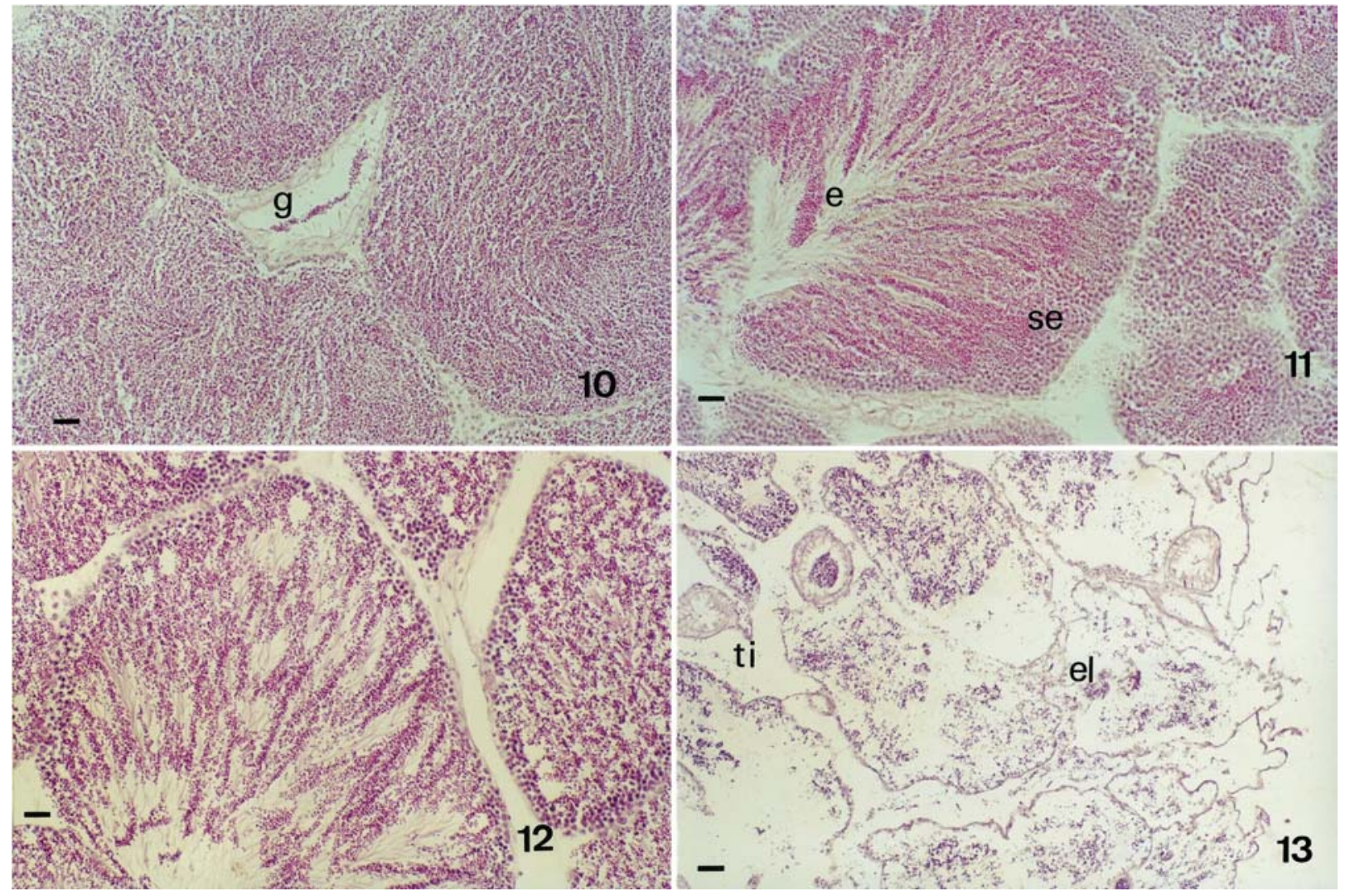

Figuras 10-13. Fases del desarrollo gametogénico del sector masculino de la gónada de Euvola ziczac: (10) maduración máxima; (11) evacuación con proliferación; (12) evacuación con agotamiento; (13) reversión. (e) Espermatozoides; (el) espermatozoides en lisis; (g) conducto eferente; (se) serie espermática; (ti) tejido interfolicular. Escala: $50 \mu \mathrm{m}$.

ros. Tanto en uno como en el otro caso, existe una intensa actividad proliferativa con la presencia de un gran número de ovogonias y ovocitos previtelogénicos en la pared del folículo (Fig. 6). Puede existir un leve aumento de tejido interfolicular, sin llegar a caracterizar una fase de reversión gonadal.

Desove total: folículos totalmente vacíos, con paredes íntegras, sin señales de actividad proliferativa o de reversión gonadal. Pocos individuos fueron encontrados en esta fase (Fig. 7).

Desove total con proliferación: existe en este caso un disparo proliferativo en las paredes de los folículos, los cuales permanecen vacíos, formando una figura semejante a un collar de perlas (Fig. 8).

Reversión: reducción drástica del diámetro de los folículos, los cuales se contraen aumentando el espacio interfolicular, donde pueden reconocerse numerosos elementos fagocíticos (amebocitos). Todas las células de la serie ovogénica en el interior de los folículos muestra señales de atresia o degeneración, con fuerte basofilia y contornos irregulares (Fig. 9).

Para el sector masculino, las fases de la escala de madurez definidas fueron:

Maduración máxima: folículos expandidos (diámetros máximos entre 500-1000 $\mu \mathrm{m}$ ), con el interior dominado por densas columnas de espermatozoides maduros. La pared folicular puede mostrar una delgada capa de elementos iniciales de la serie espermática. Pueden observarse conductos eferentes expandidos e con masas de espermatozoides en su interior indicando una evacuación inminente (Fig. 10).

Evacuación con proliferación: el diámetro de los folículos se reduce $(400-600 \mu \mathrm{m})$, con aproximadamente $75 \%$ de su interior ocupado por espermatozoides maduros alineados en columnas radiales compactas, y el resto ocupado por una gruesa capa $(20-50 \mu \mathrm{m})$ de elementos proliferativos de la serie espermática junto a la pared del folículo (Fig. 11).

Evacuación con agotamiento: los espermatozoides aparecen de forma menos densa y en columnas mas estrechas. Serie espermática presente, pero en menor abundancia y formando espacios libres entre sus componentes. Puede existir un incremento de tejido interfolicular (Fig. 12).

Reversión: como en los sectores femeninos, existe una reducción drástica del tamaño de los folículos y aumento de tejido interfolicular. Dentro de los folículos, los pocos componentes remanentes de la serie espermática muestran señales de lisis, llegando a formar aglutinaciones en forma de grumos. Los amebocitos dominan el espacio interfolicular (Fig. 13).

El análisis de la porción femenina de vieiras del banco 

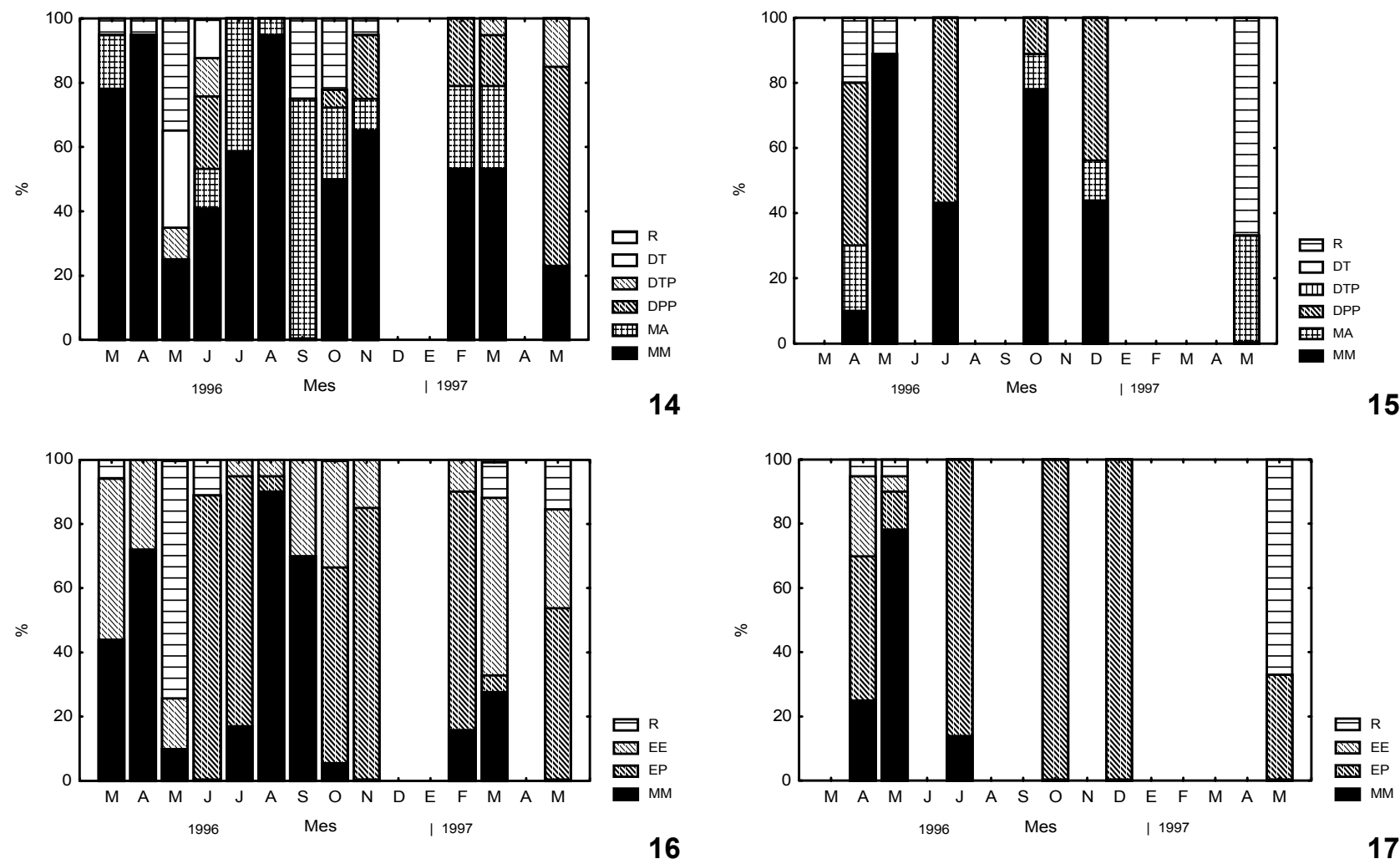

Figuras 14-17. Porcentajes, para cada mes de colecta, de las diferentes fases de la gametogénesis en la porción femenina (14-15) y en la porción masculina (16-17) de la gónada: (14 y 16) banco de São Francisco; (15 y 17) banco de Bom Abrigo. (DPP) Desove parcial con proliferación, (DT) desove total, (DTP) desove total con proliferación, (EE) Evacuación con agotamiento, (EP) evacuación con proliferación, (MA) maduración y atresia, (MM) maduración máxima, (R) reversión.

de São Francisco (Fig. 14) mostró la existencia de individuos en maduración máxima en prácticamente todos los cruceros. La presencia de desoves parciales con proliferación junto con proporciones considerables de individuos en maduración máxima indican que la liberación de gametos femeninos estaría ocurriendo prácticamente durante todo el año. Sin embargo, podemos reconocer dos períodos de mayor ocurrencia de individuos maduros (> 80\%), uno durante el final del verano e inicio del otoño (marzo-abril/96; febrero-marzo/97) y otro en el invierno (agosto/96), extendiéndose durante la primavera. Fases de desove total y de reversión ocurrieron con mayor frecuencia inmediatamente después de este período. Fases de maduración y atresia fueron observadas también en la mayoría de los cruceros, pero con una mayor ocurrencia (70\%) en septiembre/96. Sin embargo, parece existir una rápida recuperación de este fenómeno de atresia, que siempre muestra concomitantemente un disparo ovogonial. En los dos meses subsiguientes se registró un aumento considerable del número de fases de maduración máxima.

A pesar del poco material colectado en el banco de Bom Abrigo, los diagnósticos de la porción femenina indicarían la existencia de un desfazaje del ciclo gametogénico entre los dos bancos (Fig. 15 y 17). Los mayores valores de maduración má- xima fueron alcanzados en mayo/96, un mes después de que en São Francisco. En mayo/97 fue registrada una alta incidencia de reversión, la que puede haber ocurrido en São Francisco en abril/97, cuando no fue posible la colecta de material en este último banco.

En la porción masculina de las vieiras del banco de São Francisco (Fig. 16), los dos períodos de maduración máxima resultaron más evidentes de que en la porción femenina. También existiría en este caso una liberación casi continua de gametos, con altos porcentajes de individuos mostrando fases de maduración máxima y evacuación con proliferación en la mayoría de los meses. La reversión de mayo/96 afectó un mayor número de individuos.

La comparación de las fases de la gametogénesis entre una porción y otra de la gónada de un mismo individuo (Tab. I) muestra que existe total sincronización en la maduración máxima en apenas $20,65 \%$ del material analizado. El registro de un 20,32\% de fases de evacuación con proliferación junto con fases de maduración máxima en la porción femenina indica que existe en varios individuos un desfazaje, con la porción masculina liberando gametos antes que la femenina. Las fases de reversión ocurren sincrónicamente en las dos porciones, o bien asociadas a fases de agotamiento, en la mayor parte de los individuos. 

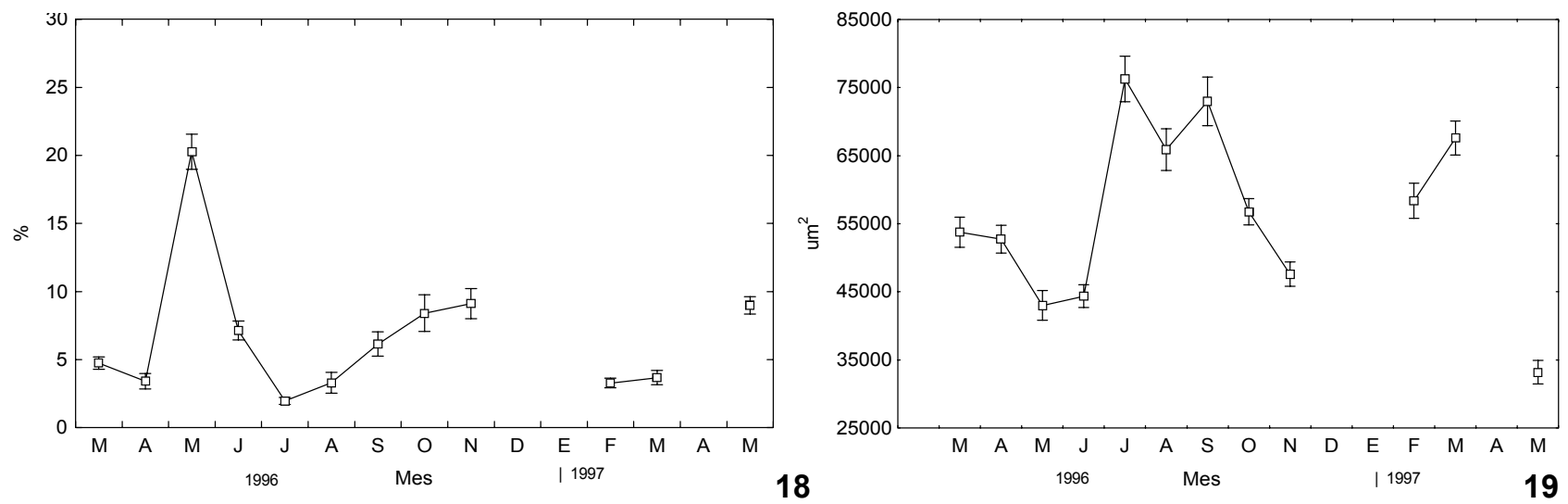

Figuras 18-19. (18) Porcentaje medio ( \pm erro padrón) y área media ( \pm erro padrón) por mes de colecta del área interfolicular de la porción femenina de individuos del banco de São Francisco.

Tabla I. Porcentaje de gónadas diagnosticadas para cada fase de la gametogénesis sobre un total de 310 individuos. (DPP) desove parcial con proliferación, (DT) desove total, (DTP) desove total con proliferación, (EE) evacuación con agotamiento, (EP) evacuación con proliferación, (MA) maduración y atresia, (MM) maduración máxima, $(\mathrm{R})$ reversión.

\begin{tabular}{crrrr}
\hline \multirow{2}{*}{ Porción masculina } & \multicolumn{4}{c}{ Porción femenina } \\
\cline { 2 - 5 } & $\mathrm{MM}(\mathrm{MA})$ & $\mathrm{DPP}+\mathrm{DTP}$ & $\mathrm{DT}$ & $\mathrm{R}$ \\
\hline MM & $20,65(5,81)$ & 0,64 & 0 & 0,97 \\
EP & $20,32(5,48)$ & 17,42 & 0,32 & 1,29 \\
EE & $5,81(3,55)$ & 4,19 & 0,32 & 3,87 \\
R & $0,97(0,64)$ & 2,58 & 1,61 & 3,55 \\
\hline
\end{tabular}

El análisis cuantitativo de la porción femenina de la gónada confirma la interpretación del ciclo reproductivo a partir del diagnóstico de las fases de maduración. El aumento del área interfolicular, con máximos en mayo/96, noviembre/96 y mayo/97 (Fig. 18), es un indicativo de la existencia de procesos de reorganización celular (reversión). El área media folicular, con sus valores máximos asociados a fases de maduración gonadal, mostró dos máximos anuales, de marzo/96 a abril/96 y de agosto/96 a octubre/96. Un nuevo período comenzaría en febrero/97, y se extendería hasta abril/97 (Fig. 19). La variación, a lo largo del año, del número de ovocitos íntegros, también estuvo asociada a los principales períodos de maduración (Fig. 20). Las bruscas caídas registradas en mayo/96 y septiembre/ 96 estarían mostrando la importancia del volumen del desove ocurrido en algún momento anterior al muestreo. La ocurrencia de ovocitos atrésicos muestra con mayor claridad un pico en julio/96, septiembre/96 y marzo/97. Finalmente, las ovogonias fueron mas numerosas en meses anteriores a los períodos de máxima maduración, como en junio/96.

Las distribuciones de frecuencias del diámetro de los ovocitos íntegros nos indica claramente la incorporación de nuevos componentes de la serie, ovocitos previtelogénicos, al proceso de maduración (Fig. 21). Esta incorporación aparece

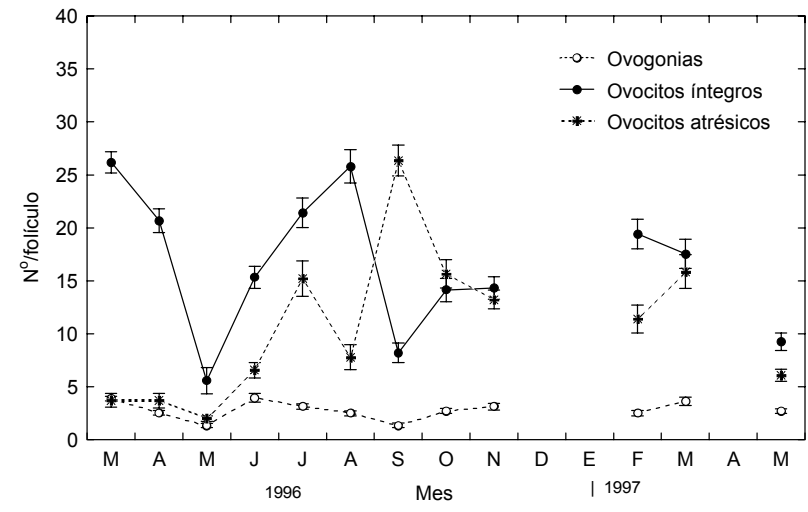

Figura 20. Número medio ( \pm erro padrón) por mes de colecta de ovogonias, ovocitos íntegros y ovocitos atresicos de individuos del banco de São Francisco.

de forma casi continua, de marzo/96 a abril/96, de junio/96 a agosto/96, y en los restantes meses analizados. La presencia de un importante disparo ovogonial durante el mes de mayo/97 sugiere la existencia de una fase de reversión o reposo previa, como mencionado anteriormente.

\section{DISCUSIÓN}

Tanto el análisis cualitativo como el cuantitativo de los cortes histológicos de la gónada de la vieira E. ziczac indican que la especie, en el área de estudio, posee una reproducción practicamente continua, con dos períodos principales de liberación de gametos, el primero a fines del verano y durante el otoño, y el segundo durante el invierno y principios de la primavera. Una de las particularidades encontrada en este estudio fue la existencia del fenómeno de la atresia de los ovocitos maduros, también denominada de lisis ovocitária. Este fenómeno, entendido como una fase de necrosis celular, fue mencionado para varias especies de bivalvos, y particularmente para miembros de la familia Pectinidae (e.g. TANG 1941,

Revista Brasileira de Zoologia 20 (4): 763-772, dezembro 2003 

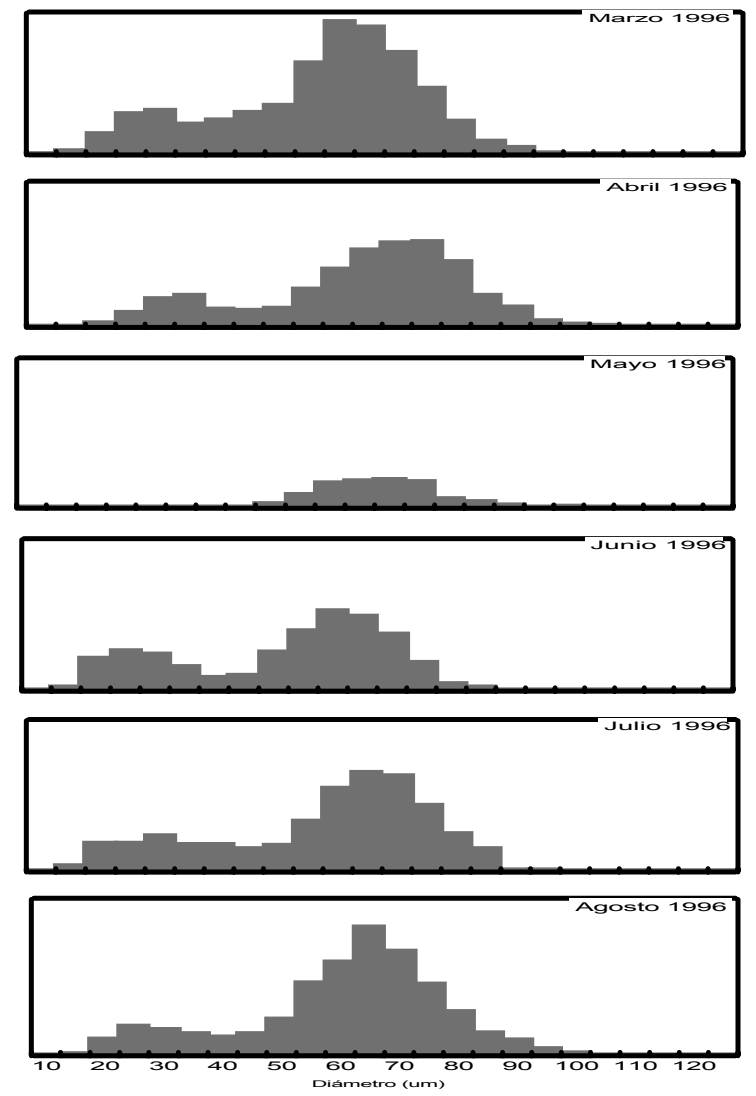

Figura 21. Distribución de frecuencias del diámetro $(\mathrm{mm})$ de ovocitos íntegros de individuos del banco de São Francisco.

Christiansen \& Olivier 1971, Ozanai 1975). En el caso específico de Euvola ziczac, este tipo particular de atresia ocurre principalmente en las fases terminales de la vitelogénesis, y debemos distinguirla de aquella que aparece en cualquier otro componente celular durante las fases de una reversión gonadal generalizada. Sin embargo, durante la necrosis de los elementos maduros son liberadas enzimas que podrían también afectar algunos ovocitos previtelogenéticos (BENINGER \& Le Penec 1991). Como fue descrito anteriormente, los ovocitos atrésicos se caracterizan por la pérdida de las propiedades basófilas del núcleo, disminución de la densidad del citoplasma y deformación periférica de la célula configurando la apariencia característica de una pieza de rompecabezas. Esta figura estuvo siempre asociada, en este estudio, a un disparo proliferativo, el cual podría ser una respuesta directa a la liberación de espacio intrafolicular, como ocurre en los desoves parciales. De hecho, muchos diagnósticos de desoves parciales podrían perfectamente corresponder a fases de maduración y atresia en estado avanzado de desintegración de los elementos maduros que no fueron efectivamente liberados por falta de un estímulo para el desove.

En Pecten maximus, la atresia ovocitaria ocurrió en épocas de máxima maduración y cuando la temperatura del agua no alcanzó valores suficientemente altos como para inducir un desove (PAulet et al. 1986). Esto mostraría que los ovocitos
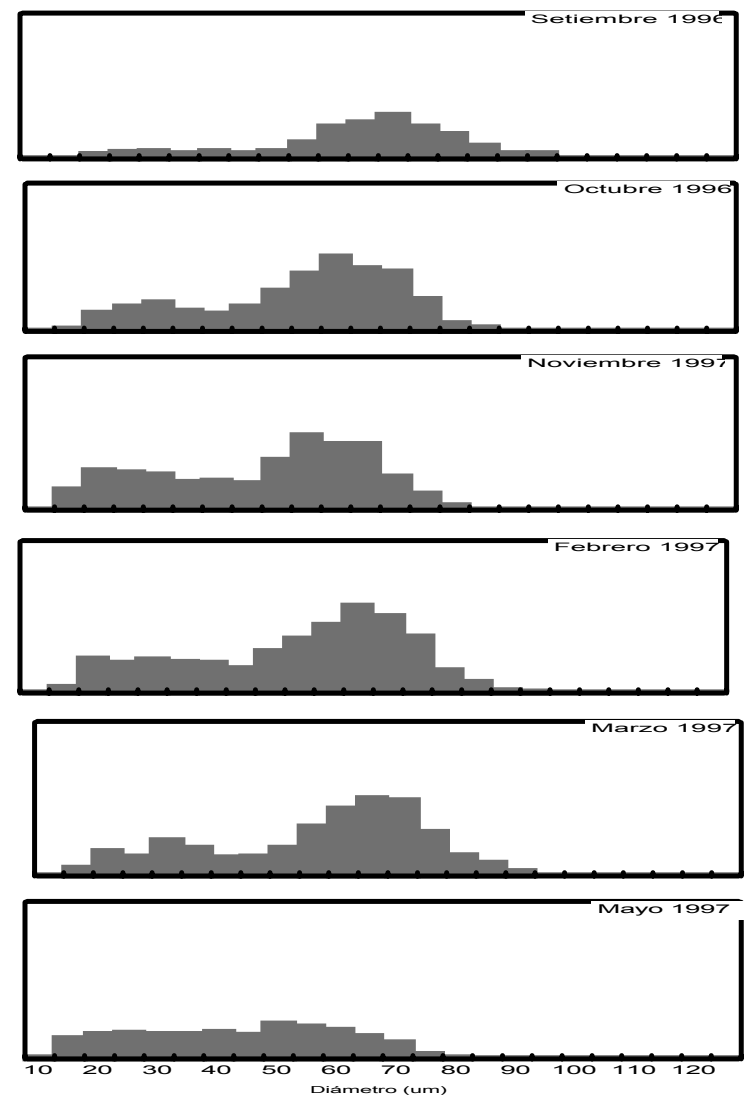

en estas especies son viables dentro de los folículos por poco tiempo, entrando en lisis celular caso no sean liberados. De esta forma, existiría un reaprovechamiento de los nutrientes existentes en el vitelo de los ovocitos y consecuentemente una optimización del proceso reproductivo cuando las condiciones de desove no son favorables (Beninger \& Le Pennec 1991, Faveris \& Lubet 1991, Lubet et al. 1991). Esta reabsorción ocurriría tanto en los folículos como en los conductos genitales (LuBEt et al. 1987 y Dorange 1989 apud Lubet et al. 1991), mostrando que la presencia de ovocitos atrésicos en los conductos no necesariamente significaría la liberación de los mismos con el consecuente desperdicio energético.

Entre los factores que estarían controlando el ciclo reproductivo de Euvola ziczac en el sur-sudeste del Brasil, la temperatura del fondo, con su ciclo semianual, parece ser el principal. Los dos períodos de mayor maduración máxima coincidieron con las elevaciones de la temperatura como consecuencia de la homogenización de la columna de agua. Por otro lado, esta elevación de la temperatura fue menor en el segundo período, durante el invierno, pudiendo ser este el motivo de la falta de estímulo para el desove y la consecuente mayor incidencia de atresia de ovocitos maduros en septiembre. El fenómeno de intrusión de la ACAS no es apenas determinante de una disminución de la temperatura del agua de fondo durante el verano, sino que también representa una importante entrada 
de nutrientes con el consecuente aumento de la producción primaria (Pezzuto et al. 1998). A pesar de que este fenómeno ha sido descrito como regular y de larga escala (Bonzone et al. 1999), es posible que variaciones en su intensidad sean responsables por la mayor o menor ocurrencia de un período de desove, y como consecuencia, de las grandes fluctuaciones en los desembarques registrados para esta especie desde el comienzo de la pesquería.

Ciclos reproductivos continuos, o con marcados períodos semianuales de liberación de gametos, fueron también descriptos para Chlamys amandi Hertlein, 1935 (Jaramillo et al. 1993), C. varia (Linnaeus, 1758) (SHAFEe 1981), Pecten papyraceus Gabb, 1873 (Salaya \& Penchaszadeh 1979), y en algunas poblaciones de Argopecten irradians (Lamarck, 1819) (BRICELJ et al. 1987, Теттеlвach et al. 1999), A. gibbus (Linnaeus, 1758) (Moyer \& Blake 1986), Pecten maximus (Mason 1957, Lubet et al. 1995), e Placopecten magellanicus (Gmelin, 1791) (Du Paul et al. 1989, Sснмiтzer et al. 1991, Dibacco et al. 1995). Este tipo de reproducción parece ser muy importante en aquellas especies de menor longevidad ya que reduce la posibilidad de una falla en el reclutamiento de la población y su consecuente desaparición, cuando la vida de los adultos reproductores es corta o limitada (Lubet et al. 1995, Tetтelbach et al. 1999).

El ciclo reproductivo de Euvola ziczac en la Venezuela, estudiado por Vélez et al. (1987) por medio del examen macroscópico de la gónada y por Lodeiros \& HimMELMan (1994) por medio de la variación temporal del índice gonadal, también mostró dos períodos principales de liberación de gametos a lo largo del año. Estos períodos son prácticamente los mismos a los encontrados en este trabajo, de abril a mayo y de agosto a setiembre. Entretanto, existen pequeñas variaciones de un año para otro, o de un local para otro, como el pequeño desfazaje descripto entre el primero y el segundo año de este estudio, y entre los bancos de São Francisco y de Bom Abrigo. En este sentido, esta especie parece ser un buen ejemplo de la existencia de un control genético de la reproducción (COCHARD \& Devauchelle 1993, Mackie \& Ansell 1993) al contrario de otras especies de pectinídeos donde fueron encontradas grandes modificaciones en las características reproductivas a lo largo de un gradiente latitudinal de distribución (BARBER \& BLAKE 1983).

La comparación de las fases del proceso gametogénico entre la porción femenina y la masculina de un mismo individuo mostró la existencia de un hermafroditismo funcional protándrico en una parte de la población. Otra parte, que tuvo maduraciones máximas en las dos porciones a un mismo tiempo, sumado a los casos de coincidencias de desoves y emisiones parciales con proliferación, correspondería a un hermafroditismo funcional simultáneo, y en este caso serían altas las posibilidades de autofecundación. Estudios genéticos preliminares llevados a cabo con material proveniente de los dos bancos estudiados, mostraron un alto grado de homozigosidad que podría ser causado por el predominio de procesos de autofecundación (WANGÜEMERT et al. 2000). Estos procesos no tendrían un efecto negativo inmediato, como fue demostrado por BETANCOURT et al. (1995) para la misma especie en experimentos de laboratorio. BEaumont \& Budd (1983) y ORENSANZ et al., (1991) señalaron que el hermafroditismo simultáneo de algunos pectinideos posee un papel fundamental en el mantenimiento de poblaciones que muestran grandes fluctuaciones en su densidad debido a fenómenos naturales o a sobreexplotación por pesca. De alguna forma, este proceso, junto con la existencia de una actividad reproductiva casi con- tinua a lo largo del año, estaría contribuyendo para mantener la existencia de la especie en la región sur-sudeste del Brasil, a pesar de la intensa pesca dirigida de años anteriores, y la probablemente continua pesca accidental de los últimos años.

\section{REFERENCIAS BIBLIOGRÁFICAS}

Aмato, J.F.R. \& S.B. Amato. 1982. Sulcascaris sulcata (Nematoda, Anisakinae) infecting sea scallops being exported from southeastern Brasil. Arquivos da Universidade Federal Rural do Rio de Janeiro. Rio de Janeiro, 5 (1): 61-67.

BARBER, B.J. \& N.J. BlaKe. 1983. Growth and reproduction of the bay scallop, Argopecten irradians (Lamarck) at its southern distributional limit. Journal of Experimental Marine Biology and Ecololgy, Amsterdam, 66: 247-256.

Beaumont, A.R. \& M.D. BudD. 1983. Effects of self-fertilization and other factors on the early development of the scallop Pecten maximus. Marine Biology, Berlin, 76: 285-289.

Beninger, P.G \& M. Le Pennec. 1991. Functional anatomy of scallops, p. 133-223. In: S.E. SHUMWAY (Ed.). Scallops: biology, ecology and aquaculture. Amsterdam, Elsevier Science Publishers, 1095p.

Betancourt, R.J.; J.E. Pérez; A. Velez; L. Freites \& M.I. Segnini. 1995. Efectos de la consanguinidad en la vieira Euvola ziczac (L). Boletin del Instituto Oceanografico de Venezuela, Universidad de Oriente, Cumana, 34 (1-2): 69-75.

Borzone, C.A. \& P.R. Pezzuto, 1997. Relatório técnico dos cruzeiros do Projeto Vieira. I. Cruzeiro I ( 4 a 9 de dezembro de 1995). Notas técnicas da FACIMAR, Itajaí, 1: 67-79.

Borzone, C.A.; P.R. Pezzuto \& E. Marone. 1999. Oceanographic characteristics of a multi-specific fishing ground of the Central South Brazil Bight. P. S. Z. N.: Marine Ecology, Nápoles, 20 (2): 131-146.

BricelJ, V.M., J. Epp \& R.E. Malouf. 1987. Intraspecific variation in reproductive and somatic growth cycles of bay scallops Argopecten irradians. Marine Ecology, Progress Series, Amelinghausen, 36: 123-137.

Castro Filho, B.M. 1990. Estado atual do conhecimento dos processos fisicos das águas da plataforma continental sudeste do Brasil. In: II Simposio de Ecossistemas da Costa Sul e Sudeste Brasileira. São Paulo, 4, p. 1-19.

Campos, E.J.D.; Y. Ikeda; B.M. Castro Filho; S.A. Gaeta; J.A. LORENZZETTI \& M.R. STE. 1996. Experiment studies circulation in the Western South Atlantic. EOS, Transactions, American Geophysic Union, San Francisco, 77 (27): 253-259.

Christiansen, H.R. \& S.R. Olivier. 1971. Sobre el hermafroditismo de Chlamys tehuelcha D'orb. 1846. (Pelecypoda, Filibranchia, Pectinidae). Anales de la Sociedad Científica Argentina, Buenos Aires, 3: 115-127.

Cochard, J.C. \& N. Devauchelle. 1993. Spawning, fecundity and larval survival and growth in relation to controlled conditioning in native and transplanted populations of Pecten maximus (L.): evidence for the existence of separate stocks. Journal of Experimental Marine Biology and Ecololgy, Amsterdam, 169: 41-56.

Dibacco, C.; G. Robert \& J. Grant. 1995. Reproductive cycle of the sea scallop, Placopecten magellanicus (Gmelin, 1791), on northeastern Georges Bank. Journal of Shellfish Research, Southampton, 14 (1): 59-69.

Du Paul, W.D.; J.E. Kirkley \& A.C. Schmitzer. 1989. Evidence of 
a semianual reproductive cycle for the sea scallop, Placopecten magellanicus (Gmelin, 1791), in the Mid-Atlantic region. Journal of Shellfish Research, Southampton, 8 (1): 173-178.

Faveris, R. \& P. Lubet. 1991. Energetic requirements of the reproductive cycle in the scallop Pecten maximus (Linnaeus, 1758) in Baie de Seine (Channel), p. 67-73. In: S. Shumway $\&$ P. A. Sandifer (Eds). An International Compendium of Scallop Biology and Culture. New York, The World Aquaculture Society, 357p.

Jaramillo, E.; J. Winter; J. Valencia \& A. Rivera. 1993. Gametogenic cycle of the chiloe scallop (Chlamys amandi). Journal of Shellfish Research, Southampton, 12 (1): 59-64.

Lasta, M. \& J. Calvo. 1978. Ciclo reproductivo de la vieira (Chlamys tehuelcha) del golfo San José. Comunicaciones de la Sociedad Malacológica del Uruguay, Montevideo, 5 (35): 1-45.

Lodeiros, C.J. \& J. Himmelman. 1994. Relations among environmental conditions and growth in the tropical scallop Euvola (Pecten) ziczac (L.) in suspended culture in the Golfo de Cariaco, Venezuela. Aquaculture, Amsterdam, 119: 345-358.

Lubet, P.; R. Faveris; J.U. Besnard; I. Robbins \& P. Duval. 1991. Annual reproductive cycle and recruitment of the scallop Pecten maximus (Linnaeus, 1758) from the Bay of Seine, p. 87-94. In: S. SHUMWAY \& P.A. SANDIFER (Eds). An International Compendium of Scallop Biology and Culture. New York, The World Aquaculture Society, 357p.

Lubet, P.; N. Devauchelle; M.L. Muzellec; Y.M. Paulet; R. Faveris \& J.C. DAO. 1995. Reproduction of Pecten maximus from different fisheries areas: Rade de Brest, Baie de Saint-Brieuc, Baie de Seine. IFREMER, Actes de Colloques, Issy-LesMoulineaux, 17: 157-163.

Mackie, L.A. \& A.D. AnSell. 1993. Differences in reproductive ecology in natural and transplanted populations of Pecten maximus: evidence for the existence of separate stocks. Journal of Experimental Marine Biology and Ecology, Amsterdam, 169: 57-75.

Mason, J. 1957. The age and growth of the scallop, Pecten maximum (L.), in Manx waters. Journal of Marine Biology Association, Cambridge, 36: 473-492.

MatsuURA, Y. 1986. Contribuição ao estudo da estrutura oceanográfica da região sudeste entre Cabo Frio (RJ) e Cabo de Santa Marta Grande (RG). Ciência e Cultura, São Paulo, 38 (8): 1439-1450.

. 1996. A probable cause of recruitment failure of the Brazilian sardine Sardinella aurita population during the 1974/75 spawning season. South Africa Journal of Marine Sciences, Cape Town, 17: 29-35.

MoraIs, C. \& M. KaI. 1980. Considerações gerais sobre o manuseio e processamento de moluscos vieiras. Boletim ITAL, Campinas, 17 (3): 253-273.

MOYer, M.A. \& N.J. BlAKe. 1986. Fluctuations in calico scallop production (Argopecten gibbus), p. 45-58. In: Texas A \& M University (Ed.). Proceedings of the Eleventh Annual Tropical and Subtropical Fisheries Conference of the Americas. Texas Agricultural Extension Service, Department of Animal Science, 304p.

Orensanz, J.M.; A.M. Parma \& O. Iribarne, 1991. Population dynamics and management of natural stocks, p. 625-713. In: S. E. Shumway (Ed.). Scallops: biology, ecology and aquacul- ture. Elsevier Science Publishers, Amsterdam, 1095p.

OZANAI, K. 1975. Seasonal gonad development and sex alteration in the scallop, Patinopecten yessoensis. Bulletin of Marine Biology Stn. Asamushi, Sendai, 15: 81-88.

Paulet, Y.M.; A. Gerard \& A. Lucas. 1986. Reproductive strategy of two populations of Pecten maximus L, p. 535. In: M. Porchet; J.C. Andries \& A. Dainhaut (Eds). Advances in Invertebrate Reproduction. Amsterdam, Elsevier Science Publishers, 568p.

Perez, J.A.A. \& P.R. Pezzuto. 1998. Valuable shellfish species in the by-catch of shrimp fishery in southern Brazil: spatial and temporal patterns. Journal of Shellfish Research, Southampton, 17 (1): 303-309.

Pezzuto, P.R. \& C.A. Borzone. 1997a. The scallop Pecten ziczac (Linnaeus, 1758) fishery in Brazil. Journal of Shellfish Research, Southampton, 16 (2): 527-532.

1997b. Relatório técnico dos cruzeiros do Projeto Vieira. II. Cruzeiros II (15 a 17 de março de 1996) e III (20 a 22 de abril de 1996). Notas Tecnicas da FACIMAR, Itajaí, 1: 81-88.

Pezzuto, P.R.; C.A. Borzone; R.L.B.E. AbrahÃo; F. Brandin \& E.C. Machado. 1998. Relatório técnico dos cruzeiros do Projeto Vieira. III. Cruzeiros IV (maio de 1996) a XIV (maio de 1997). Notas Tecnicas da FACIMAR, Itajaí, 2: 109-129.

Rios, E.C. 1994. Seashells of Brazil. Rio Grande, Editora da Fundação Universidade do Rio Grande, 492p.

Salaya, J.J. \& P.E. Penchaszadeh. 1979. Pesquería de la vieira, Pecten papyraceus (Mollusca: Bivalvia), en Venezuela. Proceedings of the Gulf and Caribbean Fisheries Institute, Cancun, 31: 105-126.

Schmitzer, A.C.; W.D. Du paul \& J.E. Kirkley. 1991. Gametogenic cycle of sea scallops (Placopecten magellanicus (Gmelin, 1791)) in the Mid-Atlantic Bight. Journal of Shellfish Research, Southampton, 10 (1): 221-228.

Shafee, M.S. 1981. Seasonal changes in the biochemical composition and calorific content of the black scallop Chlamys varia (L.) from Lanveo, Bay of Brest. Oceanologica Acta, Paris, 4 (3): 331-341.

Sunyé, P.S. \& J. SERvain. 1998. Effects of seasonal variations in meterorology and oceanography on the Brazilian sardine fishery. Fisheries Oceanography, Oxford, 7 (2): 89-100.

TANG, S.F. 1941. The breeding of the scallop (Pecten maximus L.) with a note on the growth rate. Proceedings of the Liverpool Biological Society, Liverpool, 54: 9-28.

Tettelbach, S.T.; C.F. Smith; R. Smolowitz; K. Tetrault \& S. Dumais. 1999. Evidence for fall spawning of northern bay scallops Argopecten irradians irradians (Lamark 1819) in New York. Journal of Shellfish Research, Southampton, 18 (1): 47-58.

Velez, A.; L. Freites; J. H. Himmelman; W. Senior \& N. Marin. 1995. Growth of the tropical scallop, Euvola (Pecten) ziczac, in bottom and suspended culture in the golfo de Cariaco, Venezuela. Aquaculture, Amsterdam, 136: 257-276.

Velez, A.; F. Sotillo \& J. Perez. 1987. Variación estacional de la composición química de los pectínidos Pecten ziczac y Lyropecten nodosus. Boletin del Instituto Oceanografico de Venezuela, Univiversidad de Oriente, Cumaná, 26 (1-2): 67-72.

Villalejo-Fuerte, M. \& R.I. OchoA-Baez. 1993. El ciclo reproductivo de la almeja catarina, Argopecten circularis (Sowerby, 1835) 
en relación con temperatura y fotoperíodo, en Bahía Concepción, BCS, Mexico. Ciencias Marinas, Ensenada, 19 (2): 181-202.

Wanguemert, M.G.; P.R. Pezzuto \& C.A. Borzone. 2000. Preliminary analysis of the genetic variability of two natural beds of the scallop Euvola ziczac (Linnaeus, 1758) in
Brazil. Brazilian Archives of Biology and Technology, Curitiba, 42 (2): 235-240.

Zenger, H.; E.J. Victor; J.L. Agnes \& J.G. Gueran. 1975. N/Pq. Riobaldo - Relatório dos Cruzeiros $\mathrm{N}^{\circ} 02$ e 03/75 - Pesca exploratória e comercial simulada de vieiras. CIDADE?, FAO/PNUD - SUDEPE, Base de Operações do PDP/SC, 20p.

Recebido em 23.V.2003; aceito em 02.XII.2003.

Revista Brasileira de Zoologia 20 (4): 763-772, dezembro 2003 